\title{
The Metaphorical Culturalistic Approach to Technology Assessment
}

\author{
Patricia Girrbach
}

\begin{abstract}
This paper aims to demonstrate that technology assessment (TA) must be seen in a new enhanced culturalistic way cause cultural aspects determine TA in an incremental way. Therefore, it is important to analyze how the process of TA depends on cultural influences. Moreover, it is important to show how to handle this problem due to the fact that TA aims to be objective instead of cultural determined and normative. Therefore, the objective of this paper is to create a new way of reflecting TA, to show impulses for non-normative ethics in technology assessment and to present the new enhanced metaphorical culturalistic approach (MCA) of technology assessment.
\end{abstract}

Keywords: metaphorical culturalistic approach; non-normative ethics; technology assessment

\section{INTRODUCTION}

Digitalization is the essential basis for today's industry 4.0 [1]. In this context new technologies enable a new level of production including a total transformation of processes along the entire value chain of firms. These new technologies include for example well known technologies such as Internet of Things, Smart Manufacturing or Cyber Physical Systems which are crucial for nowadays industry. Based on these new technologies industry 4.0 paves the way to a new technological age including not only the incremental transformation of production, value chains and business models but also raising the way of consumption to a new level. Since new technologies can shape whole societies, it is very important to take care of their possible future drawbacks already in the development process [5]. Therefore, the crucial question of each new technology is, are there more positive effects on society than negative. Concerning this issue technology assessment (TA) comes into play focusing especially on the chances and risks arising out of a new technology $[2,5]$. Therefore, TA influences industry 4.0, in detail the technologies used. This is caused in the reason that only if technologies are assessed as useful and not dangerous for the environment (pollution or unacceptable risks which should be averted) or the society (e.g., in terms of aspects such as the health or privacy of people that should be protected) they will be produced and applied in industry. Consequently, there is a great interdependency and a high impact of TA on technologies used in industry 4.0 (Fig. 1).

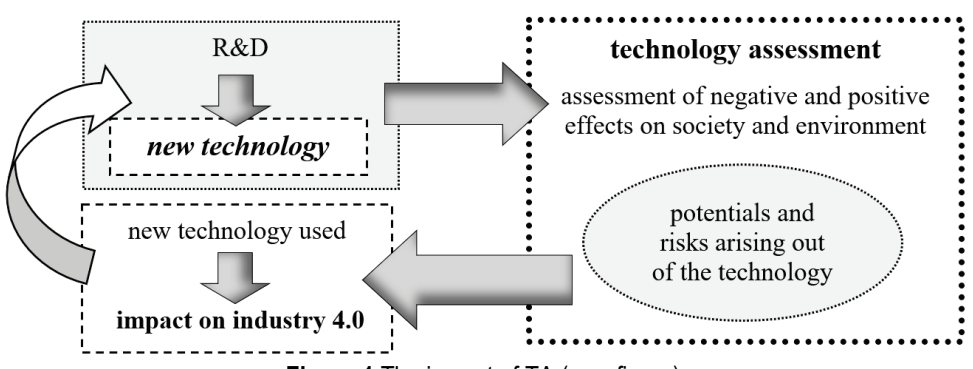

Figure 1 The impact of TA (own figure)

\section{TECHNOLOGY ASSESSMENT}

TA can be understood as the research on technology [2, $5,11]$. Hereby technology contains not only the conception, and production but also further important aspects such as the use of the technology, the disposal as well as its institutional and organizational framework conditions [3]. Moreover, TA can be considered as problem-oriented research regarding methods as well as results in the context of their actual assignment and principals $[2,5]$. Finally, TA is a scientific consulting practice offering updated well-founded orientation knowledge based on its experience with technology-induced social conflicts $[2,5,11]$. The subject is detecting and analysing not only specified goals or means but also potential un-/acceptable consequences for the society or environment rising out of a new technique $[4,5,11]$. Thus, the key question of predictive TA focuses on the consequences of technical interventions on the natural as well as the related socio-cultural environment [5]. To decide the tolerable depth of future intervention in natural cycles it is important to consider both negative un-/certain consequences of technical interventions as well as the intensity of the desired effect for given social purposes [5]. In this context various participants often rate the risks and chances of a new technology differently caused in two possible reasons [6]:

- The first reason is that participants of the TA process often start from different descriptive prerequisites, therefore their assessment in terms of actual limits, possibilities, or the specific design of future applications is different [6].

- A possible second reason can be that participants of the TA process presume various normative prerequisites; in 
this case they evaluate the expected consequences of the technology application presented based on different value systems (their normative orientation) differently [6]. It is obvious that this can lead to value conflicts during the TA process $[6,7]$.

Therefore, technology research requires not only the knowledge of the consequences of the respective interventions as part of scientific and technical risk research but also and even more important the knowledge in terms of the desirability and ethical justification of these interventions by society [5]. This includes a comprehensive debate on issues of the environmental impact (regarding environment as a kind of cultural asset) and a potential desirable change in current socio-cultural systems [5]. Thus, TA can be described as cultural science for several reasons. Especially any technology is not primarily problematic from the technical aspect of its functioning, but rather regarding its cultural significance and the specific evaluation of the consequences according to the culture and implicit cognitive models of participants. Thus, technology conflicts are determined by normative and cultural aspects such as conflicts about ideas about the future or concepts of society in an incremental way [5]. This is caused in the reason that what is socially desirable or acceptable is usually controversial depending on the cultural background of people $[6,8,13]$ (Fig. 2). Therefore, according to the socio-ecological approach of TA problems are always defined socially and in consequence culturally $[5$, $6,8]$.

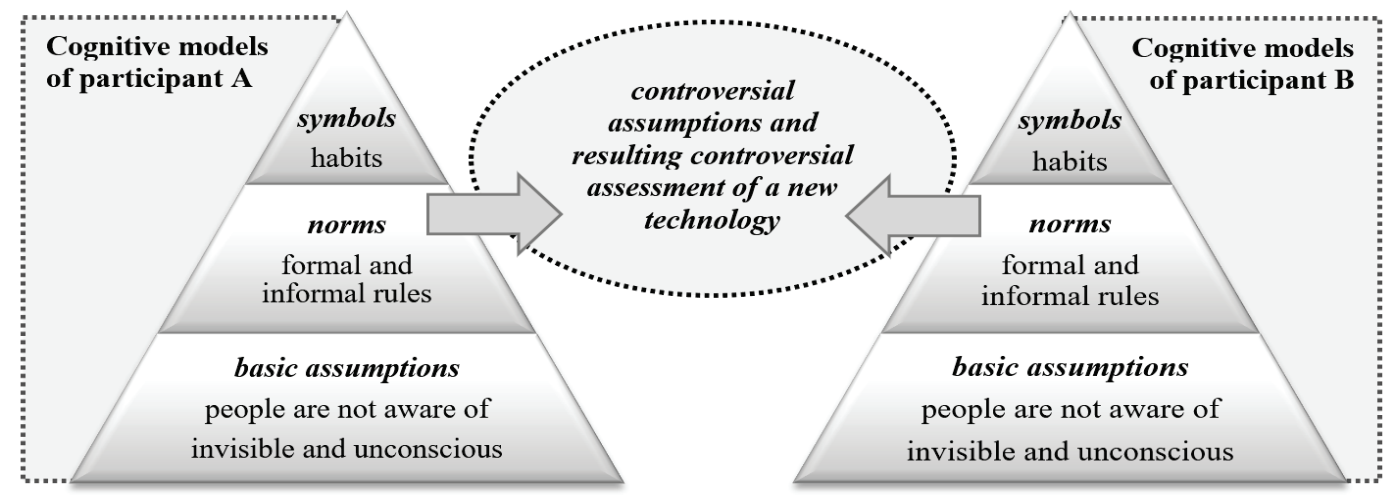

Figure 2 Controversial assumptions caused in different cognitive models (own figure based on Schein [10])

\section{INTERDEPENDENCES BETWEEN ETHICS, NORMATIVITY AND TA}

Ethics is the reflection of values and norms as well as the underlying assumptions [9]. Hereby, descriptive ethics describes and explains existing morals including formal (e.g., law) and informal rules of a society. Therefore, the subject of the research interest is the objective description \& explanation of existing values \& norms of a social group as well as the inclusion of underlying ethical principles. These underlying ethical principles are important to understand the existing morality of a society in a certain time. In contrast to that normative ethics assesses current morals and gives recommendations for improvements and better behaviour from a moral point of view [9]. In consequence normativity can be understood as a statement in which a specific evaluation is expressed [e.g., honesty is good and desirable]. In this context normative ethics tries to develop well-founded normative principles that are intended to serve as orientation and recommendation for action or regulative idea to guide individual as well as collective behaviour in a certain manner.

In addition to ecological, social, legal, or economic aspects, cultural and thus ethical and normative questions play an important role in TA $[5,6,13]$. Thus, TA is accompanied by a normative charge since normative elements as well as decisions can be identified at three levels of all TA processes $[7,13,14,15]$ :

1) The context of the TA process (e.g., presumptions, working method, selected participants of the TA process)
2) The function of TA as political advice (e.g., content of the advice, the subject such as ecological consequences or even the choice of the new technology)

3) The normative core of TA (in detail e.g., the prioritized objective of sustainability or the priority of social objectives and issues over economic ones)

So, we can identify different types of normative aspects concerning TA $[7,13,14,15]$. On the one hand there are implicit value references of TA such as in-normativity [15]. In-normativity includes values and norms of the participants taking part in the TA process. On the other hand, metanormativity comprises fundamental inherent goals of TA such as the claim for a healthy environment, the protection of privacy or sustainability as objective, as well as the oftenmentioned TA's obligation to inclusion and a democratic debate containing the well-known presumption that a more pluralistic process will always produce better outcomes than another procedure $[2,13,15,16]$. Other examples for further inherent and normative determined aspects of TA are the choice of a certain technology (the technology itself, e.g., delivery drones or robots that should be evaluated), focused areas that should be analysed in terms of the new technique as well as the selected actors involved [13].

Beside that there are also central explicit normative guiding principles of TA such as sustainability, social justice, and human rights that provide a normative framework for the assessment of new technologies [13, 16]. So, we can conclude that culture and value references determine TA in general as well as its procedures, e.g., used scientific- 
technical knowledge, concepts, goals, procedures, and consequently also the results of the TA process [13]. Accordingly, culture determines how we look at a new technology (e.g., delivery drones or robots) $[5,6]$.

Therefore, the augmented metaphorical culturalistic approach (MCA) of TA contains a new way of looking at TA. It enhances former approaches by using the metaphor of cultural glasses to point out the direct interdependence of culture in terms of our assessment. Thus, our cultural-colored glasses determine how we perceive things. Depending on the culture, the glasses have a different color. It is the same as looking at a yellow lemon with blue glasses. The result is you see the lemon green (according to traffic lights green implies e.g., the technology is accepted as useful). If your glasses are red, the lemon seems to be orange (orange implies e.g., the new technology is not accepted as useful). In this example the lemon stands for a new technology that is assessed in different ways due to different cultural assumptions (Fig. 3).

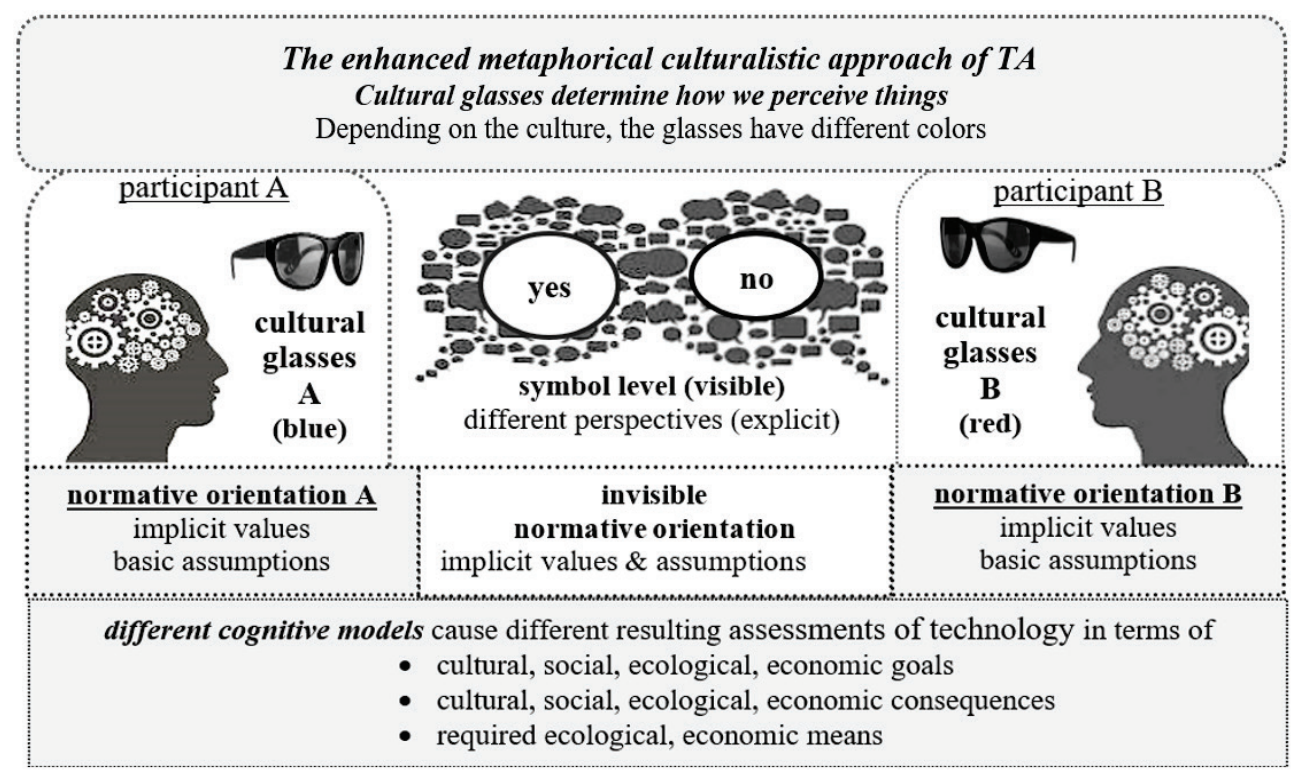

Figure 3 The enhanced metaphorical culturalistic approach of TA: Cultural glasses determine how we perceive things (own figure)

We can estimate that normativity in TA exists because it cannot be neglected that normative elements and resulting decisions can be identified at all levels and along the entire TA process [13]. In addition to ecological, social, legal, or economic aspects, cultural and thus ethical and normative questions play an important role in TA $[5,8,13]$. The crucial problem is this shatter the often-mentioned claim of TA to neutrality and non-normativity $[13,14]$. Therefore, it contradicts the TA's demand for neutrality focusing a competent, neutral assessment of the possible advantages and disadvantages of new technologies $[13,17]$. So, the question is how to deal with this existing normativity and are there any possible solutions?!

\section{FOCUSING THE INVISIBLE}

The underlying idea of the approaches shown in this paper is to replace the existing implicit normativity by reflexive normativity aiming to enable $\mathrm{TA}$ to reflect, describe, explain, and analyze the current normative structure of technology conflicts [13]. In the sense of reflexive normativity TA should provide a helpful contribution to the clarification of the normative level, including decisionmaking processes in terms of inherent goals, or the acceptability of resulting consequences for the whole society, affected people or the environment [13]. Thus, the concept of reflexive normativity does not negate normative influences of TA rather it opens the TA process and inherent normative determined assumptions for a productive discursive debate $[6,13]$. That is important since the aim is to increase procedural neutrality. Moreover, the purpose is the identification and disclosure of normative premises at all levels of TA and a systematic, method-based analysis of the value references which are relevant within each TA process. Reflexive normativity focuses on the value systems on which research is based to identify in- and explicit presuppositions for the sake of a greater transparency in terms of normative aspects [13]. Moreover, the aim is to justify the research underlying value systems with reasoned arguments for e.g., selected goals. This offers the chance and possibility to take responsibility for them. Thus, reflexive normativity aims to make values and normative preliminary decisions of TA processes transparent and more manageable [13] (Fig. 4).

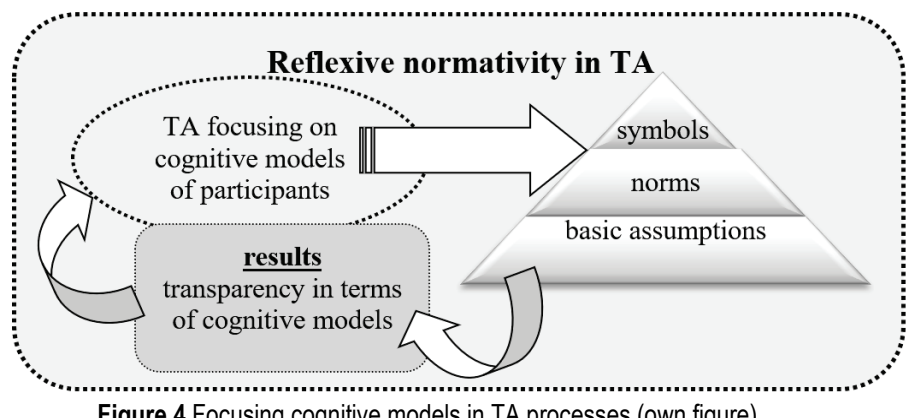

Figure 4 Focusing cognitive models in TA processes (own figure) 
So, we can summarize that the aim of reflexive normativity is to utilize the outcomes for balancing one-sided selections in favor of a greater plurality of positions and heterogeneous perspectives in TA processes $[6,13]$. Consequently, reflexive normativity replaces neutrality which is always only fictitious with the constant awareness of normative references under which TA is carried out to avoid or at least to minimize the often-unconscious selectivity in the TA process which contradicts the aim of neutrality $[13,17]$. There are several practices of reflexive normativity that can be used in the context of TA $[6,13]$ : The first starting point is to establish and operationalize reflexivity by the introduction of specific standards, rules for transparent procedures, or Codes of Conduct. Moreover, the involvement of supervisors can be useful at a very early stage who critically question the entire procedure of the project as well as underlying assumptions. So, a continuous reflection of current premises and methods along the entire process is required. Research diaries or reflexive journals can be helpful to document the research process by noting normatively relevant questions permanently and reflecting them critically, to make the path and choices made by participants more transparent, visible, and thus traceable [13]. This enhances the objectivity of the TA itself. Furthermore, the inclusion of further actors from the field of governance is recommended to change innovation trajectories in the sense of promoting the common good. To enhance the heterogeneity of opinions and to include knowledge from different areas, at best, actors such as natural, social, as well as human sciences should already communicate during development in the laboratory. Moreover, normative guiding principles and resulting ideals driving scientific research should be disclosed to support the reflection of laboratory scientists in terms on the socioethical context. Finally, the inclusion of other potentially affected parties in the process of technological development is crucial to improve and enrich the process of development of the technology itself early $[2,13,16]$. By using these methods of reflexive normativity, the transparency of cognitive models and resulting normative orientations can be enhanced. Especially in participative processes these techniques are useful to gain a broader holistic view in terms of the assessment of new technologies and to understand upcoming controversial discussions (see Fig. 5).

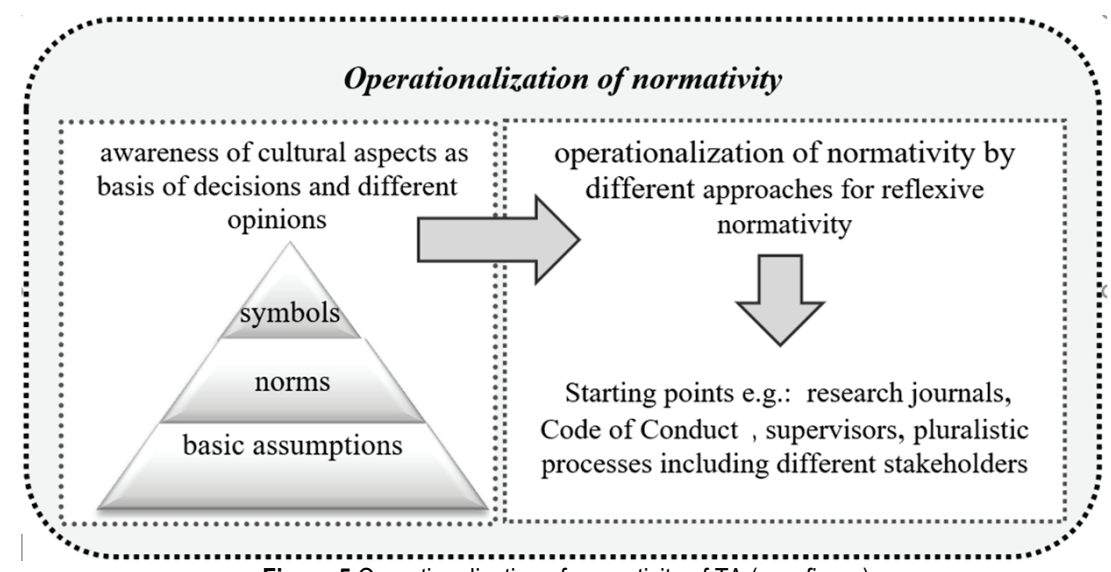

Figure 5 Operationalization of normativity of TA (own figure)

There is no doubt that participation processes are essential for reflexive normativity [6, 13]. Hereby, participants using different cognitive models, often assess differently the desirability of new technologies (e.g., robots) without recognizing why they disagree in this context [6]. This lake of understanding each other must be closed. Otherwise, an opportunity to understand each other and to reflect on one's own point of view is wasted [6]. But especially this reflection is crucial to support constructive discussions for the sake of better outcomes, to understand each other and to bring the points of different views closer together $[6,13]$. Since participants do often not have a common vocabulary with which they can explicitly express their values a common vocabulary is needed especially in participatory processes with a greater number of participants with different cultural and scientific background [6]. A common vocabulary serves as a basis for understanding each other and gaining transparency regarding the values of the participants. How this can look like, will be explained in the following chapter as part of the LOTA method [6].

\section{METHODS FOR REFLEXIVE NORMATIVITY}

The LOTA method [Landscape of Opinions for Technology Assessment] is intended to help involved participants with different background to recognize the reasons why they assess the opportunities and risks of a new technology differently [6]. The method supports discussion in participatory TA by capturing normative orientations of the participants to create transparency and to promote understanding [6]. LOTA uses a special software also called LOTA to evaluate, and to visualize data [6]. Data is collected through an online survey in which respondents answer questions about the new technology. Hereby, the selection and prioritization of the targets takes place in step 1 . This is done by answering questions on the assessment of the urgency and long-term validity of the presumed goals and on the assessment of the opportunities and risks of the technology in relation to the selected goals. Participants must subjectively assess risks and opportunities arising in their opinion for the various goals, such as safety [6]. The software 
tool evaluates the results of the research and visualizes them in diagrams. These are created for each person so that each participant can see his or her rating compared to the average of the participants of the process. Then, data is aggregated into an opinion landscape of participants that serves as a discussion starting point [6]. In the following the procedure of the LOTA method will be described.

The starting point for the online survey is a common vocabulary that serves as a basis for understanding the value foundations of the TA process [6]. But is it possible to reach agreement on a shared ethos of TA? LOTA solves this problem through an orientation towards values with already global approval. Thus, the underlying idea is to extract the vocabulary from already existing documents that have emerged from a broad-based political process [6]. In this context two important sources are for example the United Nations Sustainable Development Goals as well as the Universal Declaration of Human Rights [6]. In terms of the objectives there is no restriction to local or national contexts instead the objectives are global which implies a conscious normative setting caused in the reason that TA is understood in such a way that future consequences of a new technique (e.g., robots or drones) should be regarded for the entire planet [6]. Target systems are brought into the process; therefore, they are not normatively presupposed. Moreover, all participants have the chance to decide explicitly for or against the goals what increases transparency in terms of underlying normative models. In case participants cannot find the topic relevant to them in the given system, they even can formulate their own goal and add it to the system. In the following the different steps \& process phases of the LOTA method will be described in detail with delivery drones as new technology which has to be assessed [6].

Step 1 is an online survey: In this first step participants select and prioritize the goals and answer further questions to assess the urgency and long-term validity of the objectives as well as the opportunities and risks of the delivery drones in relation to the goals selected before [6]. Obviously, there is an abstraction gap between general global goals and the respective technology (in this case delivery drones). This gap is filled by the individual assessment of the participants without any presumed guidelines only determined by the personal underlying value systems [6].

Step 2: In the next step the evaluation of the online survey takes place where the results of the survey are visualized for each participant in radar diagrams focusing the risks and chances as well as the level of concern in terms of the different goals such as securing the basic needs [6]. To increase transparency in terms of own and other implicit assumptions participants can see the own assessment colored black and the average assessment of the others coloured green. The following figure addresses chances and risks of the new technology in detail delivery drones due to the opinions of participants (Fig. 6) [6].

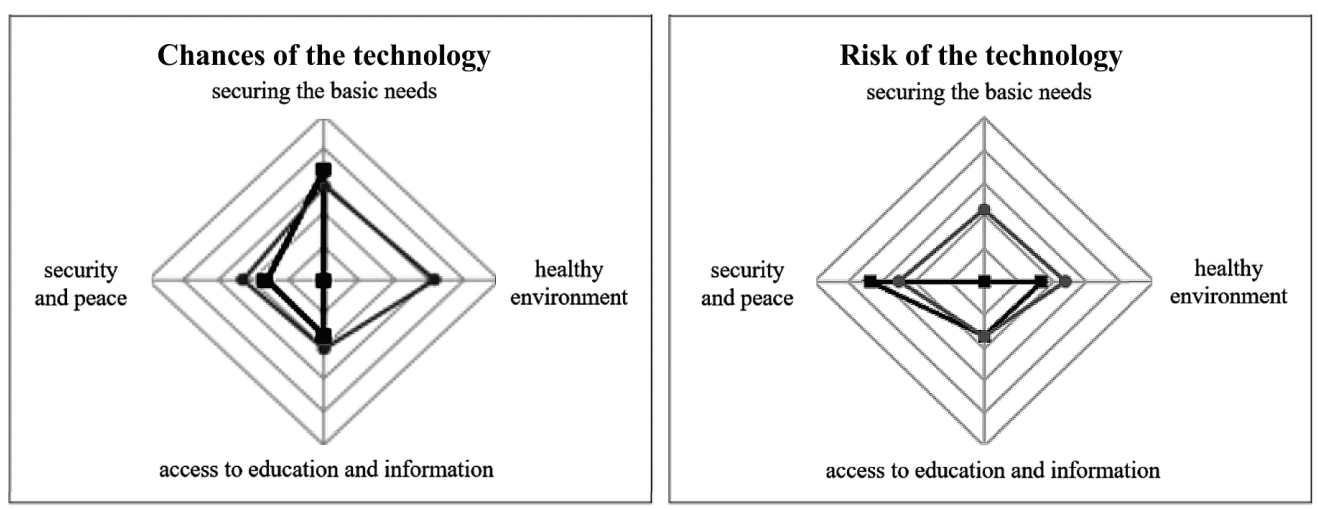

Figure 6 Radar diagrams in the TA - focusing on risks [6]

Afterwards a pseudonymized opinion landscape based on a cluster analysis is created [6]. The numerical values of the Cluster plot stand for the different participants of the survey. To save the privacy of the participants their names are pseudonymized in the Cluster plot. The moderator can use this representation to select the people to participate in the further process of TA whereby at least one person from each cluster should participate to raise the heterogeneity of different views and opinions [6]. Thus, this process increases not only the plurality of opinions, heterogeneity and diversity in the process but also promotes a more holistic view of TA.

Step 3: The third step contains a second online survey [6]: Based on the selected goals that had the highest subjective relevance for the participants the LOTA system creates a new updated list. Then all participants of the future workshop are asked once again in terms of the goals from the other actors that they had not selected themselves before.
These goals are new to the combined and updated list. Beside the questions from phase I, actors are questioned about the goals and their interaction in detail. Then, the results of step 3 are visualized as an impact graph (Fig. 7) [6].

Step 4: The impact graph is important to serve as starting point of the workshop visualizing the following information based on the opinion of the participants [6]: Target system and relevance of the targets (node size), mutual influence (arrows), influence of the technology discussed, strength of influence (arrow thickness), and standard deviation via brightness. Thus, this impact graph can be understood as a visualization of the current opinion landscape of the participants of the TA process. Since it visualizes the existing heterogeneity of opinions it creates a greater awareness of the diversity of opinions due to different normative backgrounds and supports transparency as well as a common understanding during the TA process [6]. 


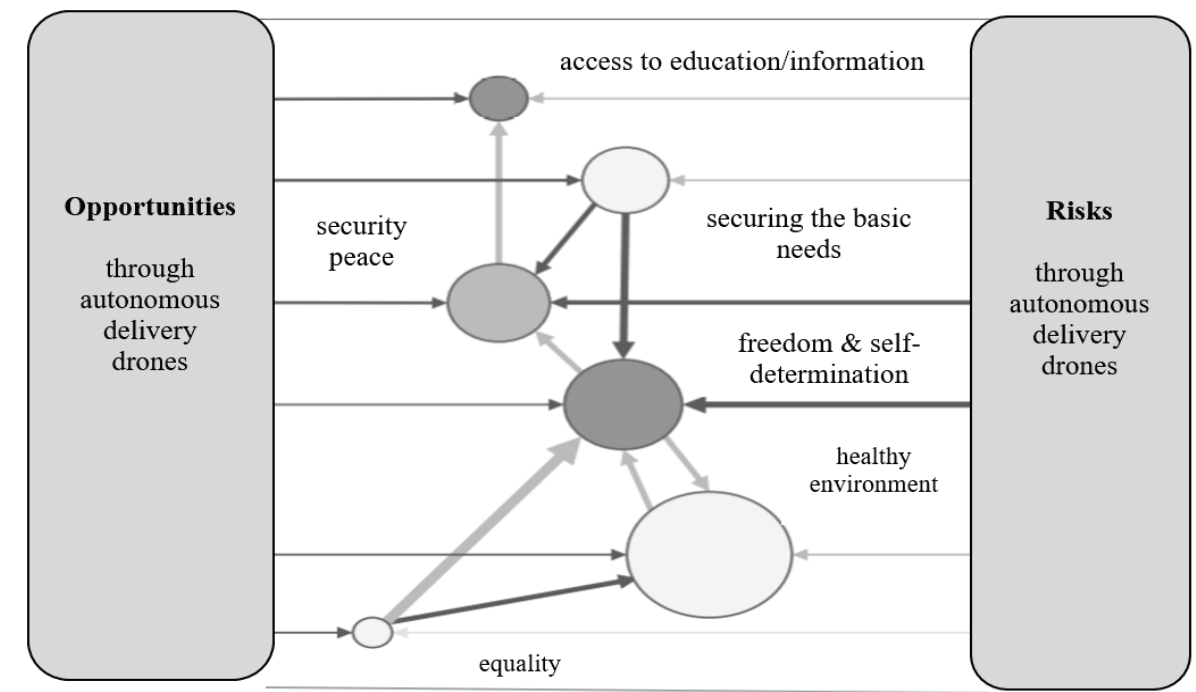

Figure 7 Impact graph (based on [6])

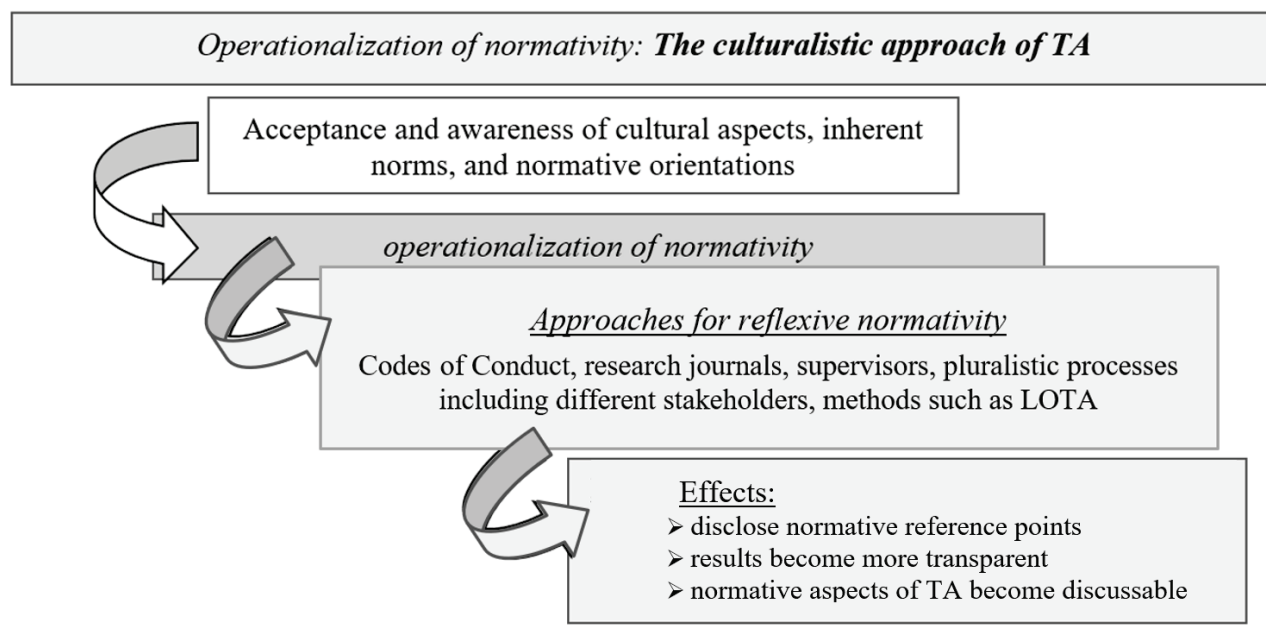

Figure 8 The culturalistic approach of TA (own figure)

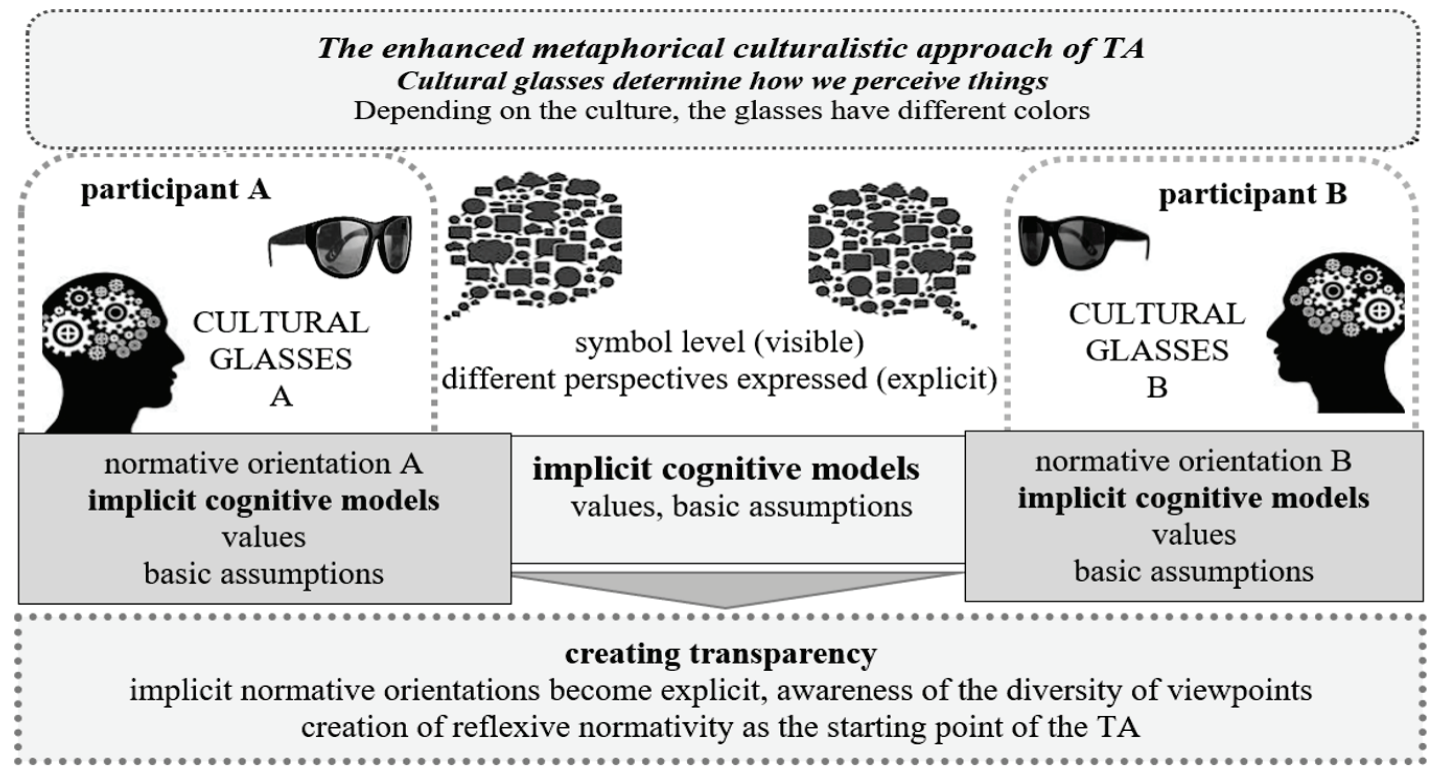

Figure 9 The enhanced metaphorical culturalistic approach (MCA) (own figure) 
Participatory TA projects bring together people not only with different scientific background but even more important with different normative orientations caused in the heterogeneity of people [6]. Therefore, it is important to face this problem and to handle it by constructive procedures such as the described LOTA method [6]. In this context we can state that LOTA serves as a methodological approach aiming to support participants in dealing with the plurality of values in a transparent and traceable way [6]. Therefore, LOTA discloses different views, creates transparency and understanding for other opinions and assessments, and prepares participants for the following discussion. Although global goals as a normative frame of reference are presented the participants cannot only express their normative orientations by prioritizing presumed goals but even enhance them due to their own normative orientation [6]. Since a landscape of opinions pointing out different views is created, the LOTA method operationalizes normativity, discloses normative reference points and makes results more transparent for all participants [6]. Consequently, inherent normative aspects of TA become discussable. This significantly increases the quality of the TA process. Therefore, the LOTA method serves as a practical tool to put the metaphorical culturalistic approach (MCA) into practice (Fig. 8 \& 9).

\section{CONCLUSION}

Today we are facing an incremental digital revolution based on new technologies offering chances and risks for the whole society including global consequences [1]. In these times TA becomes even more important than ever. Therefore, the aim of this paper is to point out an existing incremental issue in terms of TA in detail the claim for neutrality $[13,17]$. This contains neutrality and objectiveness in terms of normative and thus cultural aspects. TA focuses the assessment of new technologies (e.g., robots) in terms of arising chances and risks for the environment and the consequences for the society $[5,11]$. Moreover, TA is established to provide orientation. So, as it is pointed out in this paper, it is not even an option but a necessity to focus on normative aspects driving and controlling TA including techniques themselves, the goals to protect and many further dimensions [13]. Thus, this paper shows a concept that is based on the idea of the socio-ecological approach of TA including the insight that problems are always defined socially and culturally [5]. The cultural background consisting of various basic assumptions and resulting in/formal norms determines how people look at things such as new technologies $[5,6,13]$. This is caused in the reason that the assessment takes place based on different cognitive models that are implicit and often unconscious.

In this context the metaphorical culturalistic approach $(M C A)$ of TA is presented containing a new way of looking at TA enhancing former approaches by using the metaphor of cultural glasses pointing out the direct interdependence of culture in terms of our assessment (Fig. 9). The enhanced metaphorical culturalistic approach presented in this paper states that each cultural orientation can be seen as coloured glasses which determine our view of things we look at in an incremental way. Hereby the importance of cultural aspects is pointed out since people are always determined directly in an incremental way by their cultural orientation like wearing cultural glasses. This requires dropping out holding on the idea of neutrality of TA and substitute that view with a new metaphorical culturalistic way of looking at TA.

In this context reflexive normativity comes into play including different methods and approaches such as e.g., the introduction of codes of conduct or rules of practice [13]. Moreover, creating standards aims to establish and to operationalize reflexivity in TA practice, too. Beside that the involvement of supervisors who critically question the assumptions of the project can be useful for the sake of more reflexivity. Reflexivity along the entire process can thus be implemented [13]. By noting normatively relevant questions and reflecting them critically research diaries as well as reflexive journals document the research process [13]. Furthermore, an important aspect is to increase the heterogeneity of TA for the sake of a more holistic view. Therefore, the early inclusion of further actors from the field of governance, natural, social, and human sciences is important to address different social norms and values in a very early stage $[13,18]$. Then, normative ideals that drive scientific research can be focused, analysed, and disclosed. Consequently, the reflection of socio-ethical aspects as well as the plurality of opinions during the TA process is supported $[2,13,16,18]$. A very useful method to implement such a reflexive normativity is the LOTA method [6].

The most important point of today's TA is focusing on cultural and in consequence normative aspects, instead of neglecting them because normative aspects can be found in the whole TA process [13]. Then TA becomes not normative neutral (that is impossible since humans are part of it) but aware of its implicit normativity. That offers the chance, to change implicit to explicit reflexive normativity and to enhance the transparency in terms of TA [13].

In this context the metaphorical culturalistic approach (MCA) presented in this paper offers an incremental and crucial new insight presented as metaphor. The most important insight is understanding that each person has implicit cultural assumptions understood as cultural glasses with different color which determine how we look at things as well how we assess things in detail e.g., new technologies.

\section{Notice}

The paper was presented at MOTSP $2021-12^{\text {th }}$ International Conference Management of Technology - Step to Sustainable Production, which took place in Poreč/Porenzo, Istria (Croatia), on September 8-10, 2021. The paper will not be published anywhere else. 


\section{REFERENCES}

[1] Neugebauer, R. (2018). Digitalisierung: Schlüsseltechnologien für Wirtschaft \& Gesellschaft, Springer. https://doi.org/10.1007/978-3-662-55890-4

[2] Grunwald, A. (2019). Technology assessment in practice and theory, Routledge. https://doi.org/10.4324/9780429442643

[3] Kornwachs, K. (2018). Technikfolgenabschätzung als technikwissenschaftliche Disziplin? Methodenmix und Modellbildung. TATuP - Zeitschrift für Technikfolgenabschätzung in Theorie und Praxis, 27(1), 46-51. https://doi.org/10.14512/tatup.27.1.46

[4] Decker, M. (2007). Praxis und Theorie der Technikfolgenabschätzung. Erste Überlegungen zu einer methodischen Rekonstruktion. TATuP, 16(1), 25-34. https://doi.org/10.14512/tatup.16.1.25

[5] Renn, O. (2013). Auf dem Weg zu einer sozialökologischen Fundierung der Technikfolgenabschätzung. Simonis, G. (edt.), Konzepte und Verfahren der Technikfolgenabschätzung, 2130. https://doi.org/10.1007/978-3-658-02035-4_2

[6] Mader, C., Hilty, L., Som, C., \& Wäger, P. (2019). Transparenz normativer Orientierungen in partizipativen TA-Projekten: ein Software-basierter Ansatz. TATuP - Zeitschrift für Technikfolgenabschätzung in Theorie und Praxis, 28(1), 5864. https://doi.org/10.14512/tatup.28.1.58

[7] Weydner-Volkmann, S. (2019). Ethische TA als Kartographie situativer Wertungskonflikte. TATuP - Zeitschrift für Technikfolgenabschätzung in Theorie und Praxis, 28(1), 3944. https://doi.org/10.14512/tatup.28.1.39

[8] Hennen, L. (2019). Max Weber revisited: Die Wertbeziehung(en) der Technikfolgenabschätzung. TATuP Zeitschrift für Technikfolgenabschätzung in Theorie und Praxis, 28(1), 27-32. https://doi.org/10.14512/tatup.28.1.27

[9] Düwell, M., Hübenthal, C., \& Werner, M. (2002). Handbuch Ethik, Stuttgart, 25-35. https://doi.org/10.1007/978-3-476-02713-9

[10] Schein, E. (2003). Organisationskultur. The Ed Schein Corporate Culture Survival Guide. Fatzer, G.

[11] Grunwald, A. (2007). Auf dem Weg zu einer Theorie der Technikfolgenabschätzung: der Einstieg. Einführung in den Schwerpunkt. TATuP - Zeitschrift für Technikfolgenabschätzung in Theorie und Praxis, 16(1), 4-17. https://doi.org/10.14512/tatup.16.1.4

[12] Hennen, L. (2019). Max Weber revisited: Die Wertbeziehung(en) der Technikfolgenabschätzung. TATuP Zeitschrift für Technikfolgenabschätzung in Theorie und Praxis, 28(1), 27-32. https://doi.org/10.14512/tatup.28.1.27

[13] Kollek, R. (2019). Implizite Wertbezüge in der Technikfolgenabschätzung: Plädoyer für eine Praxis der reflexiven Normativität. TATuP - Zeitschrift für Technikfolgenabschätzung in Theorie und Praxis, 28(1), 1520. https://doi.org/10.14512/tatup.28.1.15

[14] Torgersen, H. (2018). Die verborgene vierte Dimension. TATuP - Zeitschrift für Technikfolgenabschätzung in Theorie und Praxis, 27(1), 21-27. https://doi.org/10.14512/tatup.27.1.21

[15] Nierling, L. \& Torgersen, H. (2019). Normativität in der Technikfolgenabschätzung. Einleitung in das TATuP-Thema. TATuP - Zeitschrift für Technikfolgenabschätzung in Theorie und Praxis, 28(1), 11-14. https://doi.org/10.14512/tatup.28.1.11

[16] Moser, E. (2018). Normative Leitbilder in der Technikfolgenabschätzung. ITA-manu:script, $\mathrm{Nr}$.: ITA-18-02 http://epub.oeaw.ac.at/ita/ita-manuscript/ita_18_02.pdf
[17] Bimber, B. (1996). The politics of expertise in congress. The rise and fall of the Office of Technology Assessment, State University of New York Press.

[18] Sutcliffe, H. (2000). A Report on Responsible Research and Innovation. MATTER and the European Commission, https://ec.europa.eu/programmes/horizon2020/sites/default/fil es/rri-report-hilary-sutcliffe_en.pdf

\author{
Author's contacts: \\ Patricia Girrbach, Professor Doctor \\ FOM, Fachhochschule für Ökonomie and Management, \\ Rotebühlstraße 121, 70178 Stuttgart, Germany \\ Tel.: (+49) 8001959595 \\ p.girrbach@gmx.de
}

